\title{
AVALIAÇÃO DO PH EXTERNO RADICULAR DO HIDRÓXIDO DE CÁLCIO ASSOCIADO A DIFERENTES FÁRMACOS
}

\section{EVALUATION OF THE EXTRARADICULAR PH OF CALCIUM HYDROXIDE IN ASSOCIATION WITH VARIOUS DRUGS}

\author{
Maria Carolina da Motta Rodrigues* \\ Luiz Felipe de Oliveira Rangel** \\ Vânia Filippi Goulart Carvalho Pereira"* \\ Elaine de Sá Chaves"*** \\ Joaquim Carlos Fest da Silveira*****

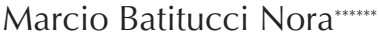

\begin{abstract}
RESUMO
Introdução: Este estudo foi realizado na Universidade Severino Sombra, onde se avaliou o pH externo radicular do hidróxido de cálcio associado a diferentes fármacos. Métodos: Foram utilizados 20 dentes humanos divididos em dois grupos de 10 dentes. Grupo I - associação do hidróxido de cálcio com trietanolamina; Grupo II - hidróxido de cálcio associado a anestésico. Os grupos foram preparados e preenchidos com as pastas. Foram imersos em água destilada e o pH medido nos tempos de 0, 24, 48, 72 horas e 7, 10, 15 e 30 dias. Resultados: Os dados obtidos foram analisados estatisticamente e o resultado mostrou diferenças significativas; observouse que em todos os tempos houve aumento do $\mathrm{pH}$ e que o $\mathrm{pH}$ mais elevado ocorreu no Grupo I. Conclusão: Concluiu-se que o hidróxido de cálcio associado à trietanolamina apresentou um pH mais elevado quando comparado ao anestésico.

DESCRITORES: Endodontia • Hidróxido de Cálcio • Anestésicos locais • Trietanolamina.
\end{abstract}

\section{ABSTRACT}

Introduction: This study was conducted at the Severino Sombra University, evaluating the extraradicular $\mathrm{pH}$ of calcium hydroxide in association with various drugs. Methods: Using twenty human teeth divided into two groups of ten teeth each. Group I - association of calcium hydroxide with triethanolamine; Group II - calcium hydroxide associated with anesthetic. The Groups were prepared and filled with the pastes, and were then immersed in distilled water, measuring the $\mathrm{pH}$ at intervals of $0,24,48,72$ hours and 7, 10, 15 and 30 days. Results: The resulting data were analyzed statistically and the findings presented significant differences, with an increase in the $\mathrm{pH}$ at all intervals, and the highest $\mathrm{pH}$ noted in Group I. Conclusion: This led to the conclusion that calcium hydroxide associated with triethanolamine presented a higher $\mathrm{pH}$ compared to the anesthetic.

DESCRIPTORS: Endodontics, Calcium hydroxide, Local anesthetics, Triethanolamine.

\footnotetext{
* Graduada em Odontologia pela Universidade Severino Sombra, Vassouras/RJ, Brasil.

** Professor Mestre em Endodontia pela Universidade de Taubaté, São Paulo, Brasil.

*** Professora Doutora em Biologia Animal pela UFRRJ, Rio de Janeiro, Brasil.

**** Professora Mestre em Ensino de Ciência da Saúde pela UNIFOA, Volta Redonda/RJ, Brasil.

***** Professor Mestre em Endodontia, pela Universidade de Taubaté, São Paulo, Brasil.

****** Professor Mestre em Endodontia, pela Universidade de Taubaté, São Paulo, Brasil.
} 
RODRIGUES MCM

RANGEL LFO

PEREIRA VFGC

CHAVES ES

SILVEIRA JCF

NORA MB

AVALIAÇÃO DO

PH EXTERNO

RADICULAR DO

HIDRÓXIDO DE

CÁLCIO ASSOCIADO

A DIFERENTES

FÁRMACOS
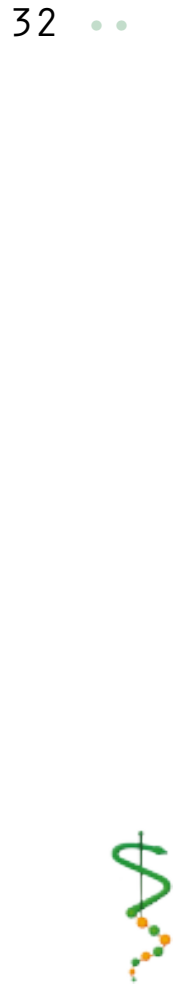

REV. ODONTOL

UNIV, CID, SÃO

PAULO

2013; 25(1): $31-9$,

$J A N-A B R$

\section{N T R O D U ÇÃ O}

Hoje uma das grandes preocupações em endodontia é conseguir promover a completa limpeza dos canais radiculares, sendo que a presença dos microrganismos é um fator responsável pela presença de patologias pulpares e periapicais, e com isso podendo ocorrer insucesso no tratamento endodôntico.

Para o sucesso no tratamento desses canais radiculares, a redução desses microrganismos é muito importante, essa diminuição está ligada a vários fatores como um preparo biomecânico e o químico mecânico bem executado. Uma das principais finalidades do tratamento endodôntico consiste na redução ou eliminação da microbiota intracanal.

Em alguns casos é necessário fazer o uso de medição intracanal entre uma sessão e outra, com o objetivo de combater microrganismos que resistiram à sanificação dos canais e com isso favorecer o processo de reparo periapical. Para ser uma boa medicação intracanal, esta tem que ser biocompatível, antimicrobiana e estimular a reparação tecidual.

O hidróxido de cálcio possui algumas propriedades, como: ação anti-inflamatória, ação antibacteriana, biocompatibilidade, solvente de matéria orgânica, inibição da reabsorção e indução de reparo mineralizado, dado que ele possui o pH elevado. Com isso o hidróxido de cálcio está indicado como medicação intracanal na prática endodôntica entre sessões tanto para caso de polpa viva como polpa necrosada e/ou com presença de lesões periapicais ${ }^{1}$.

A ação do hidróxido de cálcio está relacionada à sua dissociação em íons hidroxila e íons de cálcio e à consequente mudança do pH do meio. É necessário que ocorra difusão dos íons hidroxila através dos túbulos dentinários para que haja mudança no pH da superfície externa radicular. Além da permeabilidade dentinária e da capacidade tampão da dentina, outro fator que pode interferir na capacidade de difusão do hidróxido de cálcio são os veículos utilizados.

Para conseguir resultado satisfatório no tratamento nos casos de infecções há ne- cessidade da manutenção da alcalinização do meio. Por isso, vários estudos com novas substâncias que possam melhorar e manter o pH alcalino são importantes dentro do tratamento endodôntico.

Sendo assim, mostrou-se oportuno avaliar o pH externo radicular após a utilização de associações de hidróxido de cálcio com diferentes fármacos, como o anestésico e a trietanolamina.

\section{MÉ T ODOS}

O presente estudo foi realizado na intenção de se avaliar o pH do hidróxido de cálcio associado a anestésico e à trietanolamina e utilizado como medicação intracanal. Dessa forma, o projeto de pesquisa foi submetido ao Comitê de Ética e Pesquisa da Universidade Severino Sombra, sob o número 014/2012-01, e aprovado no dia 24 de maio de 2012.

Foram selecionados 20 dentes caninos humanos extraídos, obtidos do banco de dentes da Universidade Severino Sombra. Os dentes foram divididos em dois grupos de 10 cada, lavados em água corrente, desinfetados e reidratados por 72 horas em solução fisiológica.

A seguir, foi realizada cirurgia de acesso com uma ponta diamantada esférica 1015 e uma tronco-cônica 3181, ambas da marca KG SORENSEN e esvaziamento do canal com uma lima fina $\# 10$ e hipoclorito de sódio a 2,5\%.

O comprimento de trabalho foi obtido pelo método de visualização, introduzindo-se uma lima KEER \#15 medindo o comprimento do dente e subtraindo-se $1 \mathrm{~mm}$. A instrumentação nas porções média e cervical foram as brocas Gates-Gliden $n^{\circ} 1,2,3$. E a intrumentação seriada foi até a lima de número 50 como último instrumento do preparo. Realizou-se a irrigação com hipoclorito de sódio a 2,5\% e a irrigação final com $5 \mathrm{ml}$ de EDTA-T a $17 \%$, permanecendo 3 minutos dentro do canal. Em seguida, procedeu-se à secagem dos condutos com cones de papel absorvente de número 50, marca DENTSPLY.

Os dentes foram impermeabilizados no terço cervical e médio com uma demão de esmalte incolor para unhas, marca Colorama. Na sequência, foi manipulado o silicone de condensação, pasta pesada 
marca SPEDEX, e inserido os dentes para que não ocorresse o extravasamento da medicação.

Em seguida foram prenchidos os canais dos dentes com as medicações pesquisadas:

- O Grupo I com 10 dentes, preenchidos os canais radiculares com hidróxido de cálcio PA 10,1120g associado a anestésico 11,0701g manipulado com uma consistência que permitiu o prenchimento com uma broca lentulo e selado com um cimento provisório Cotosol marca VIGODENT.

- O Grupo II com 10 dentes, preen-

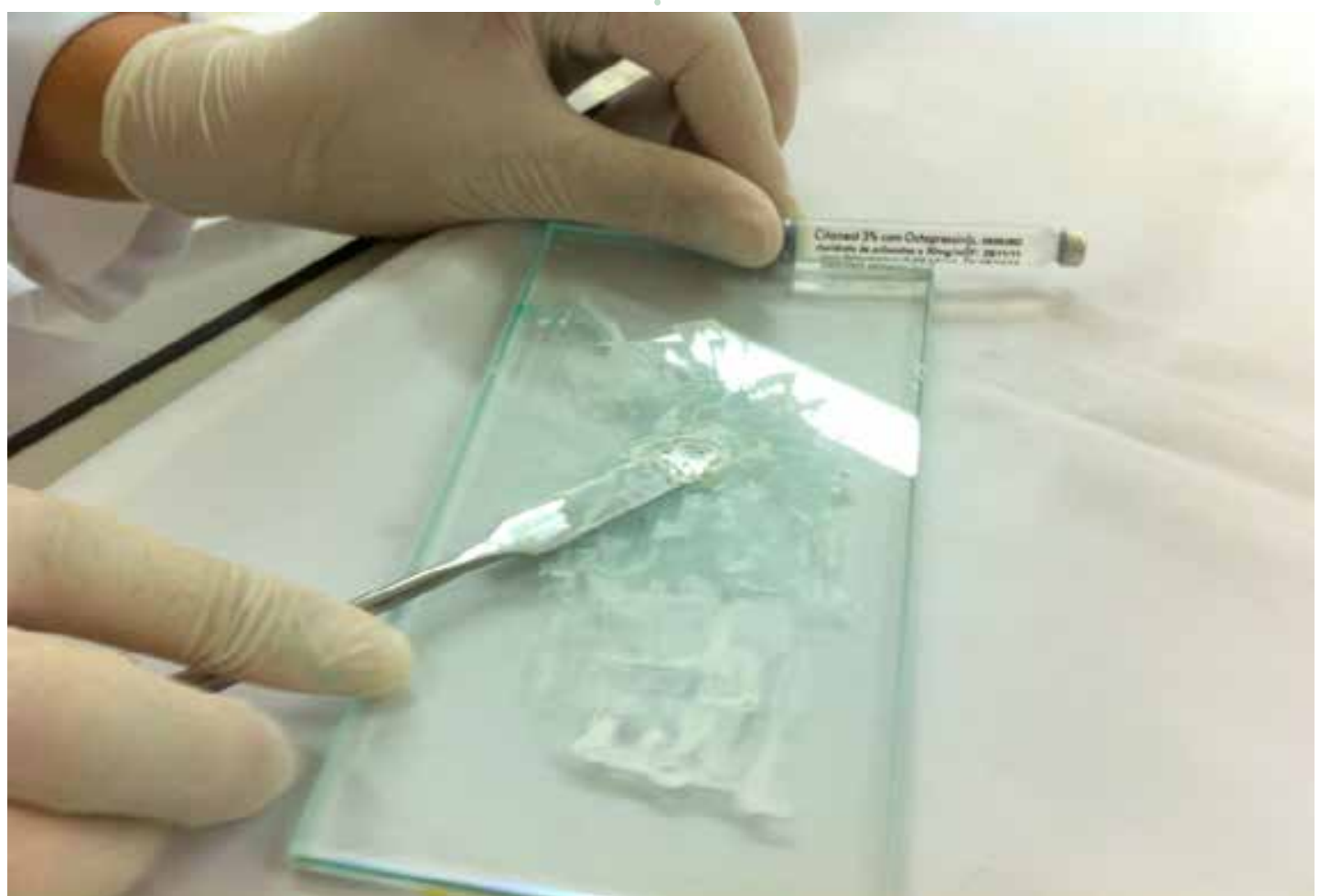

Figura 1 - Hidróxido de cálcio associado a anestésico

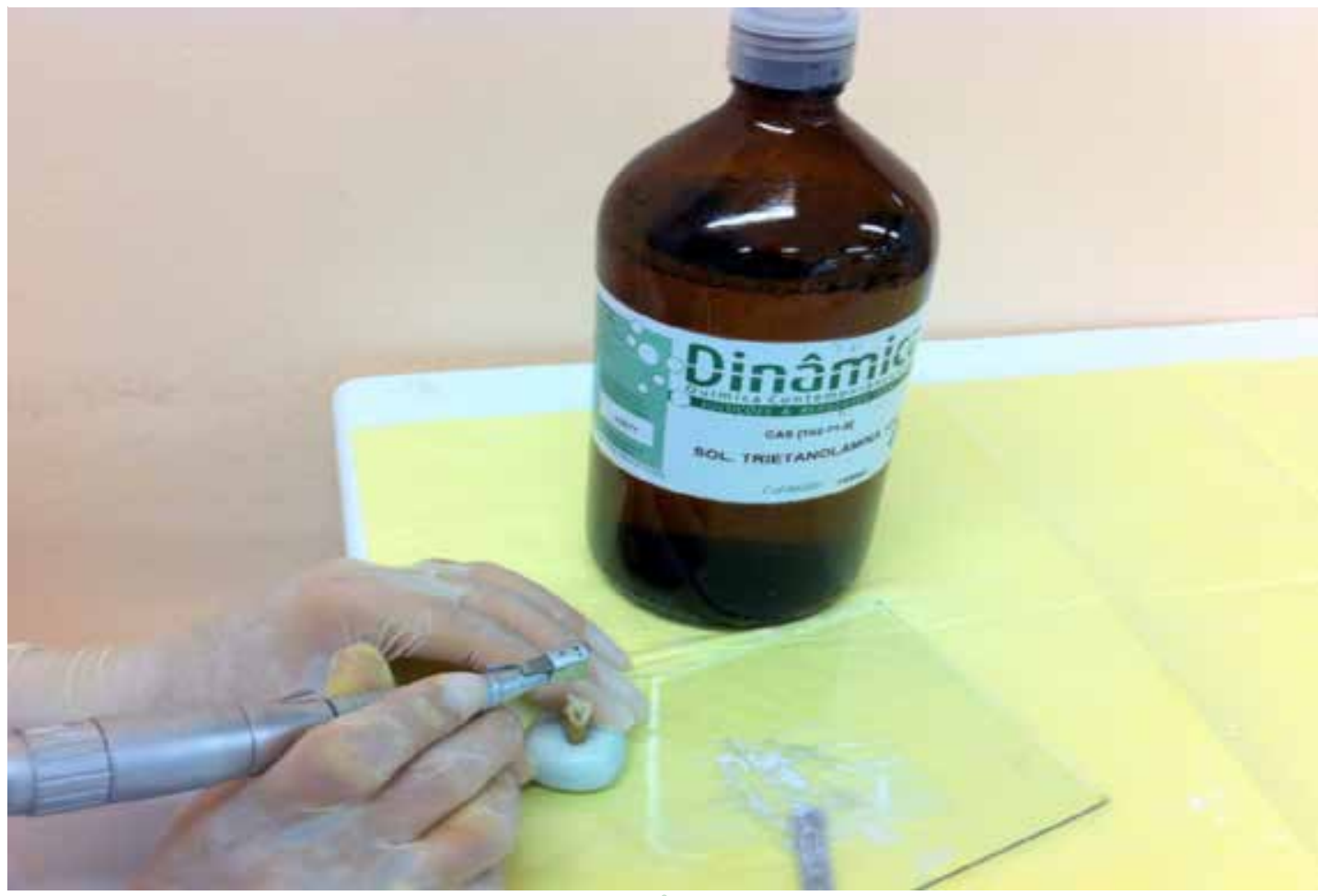

Figura 2 - Hidróxido de cálcio associado à trietanolamina
RODRIGUES MCM

RANGEL LFO

PEREIRA VFGC

CHAVES ES

SILVEIRA JCF

NORA MB

AVALIAÇÃO DO

PH EXTERNO

RADICULAR DO

HIDRÓXIDO DE

CÁLCIO ASSOCIADO

A DIFERENTES

FÁRMACOS

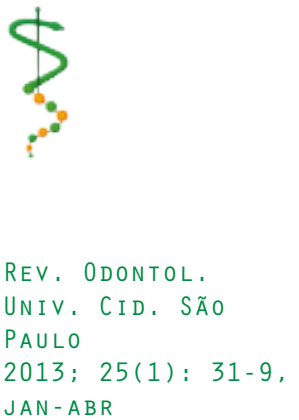


RODRIGUES MCM :

RANGEL LFO

PEREIRA VFGC

CHAVES ES

SILVEIRA JCF

NORA MB

AVALIAÇÃO DO

PH EXTERNO

RADICULAR DO

HIDRÓXIDO DE

CÁLCIO ASSOCIADO

A DIFERENTES

FÁRMACOS

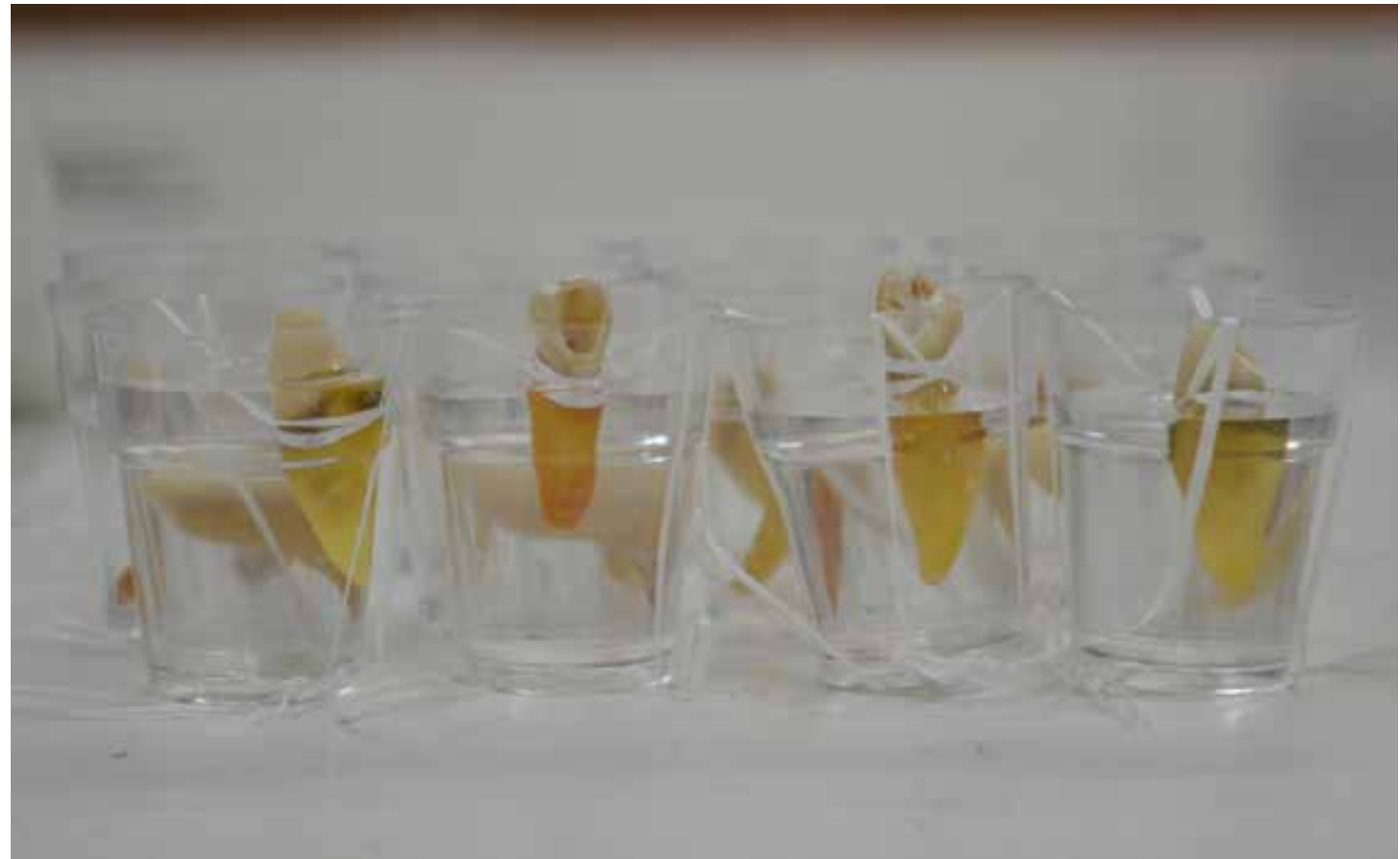

Figura 3 - Dentes nos recipientes para ser feita a mensuração do $\mathrm{pH}$

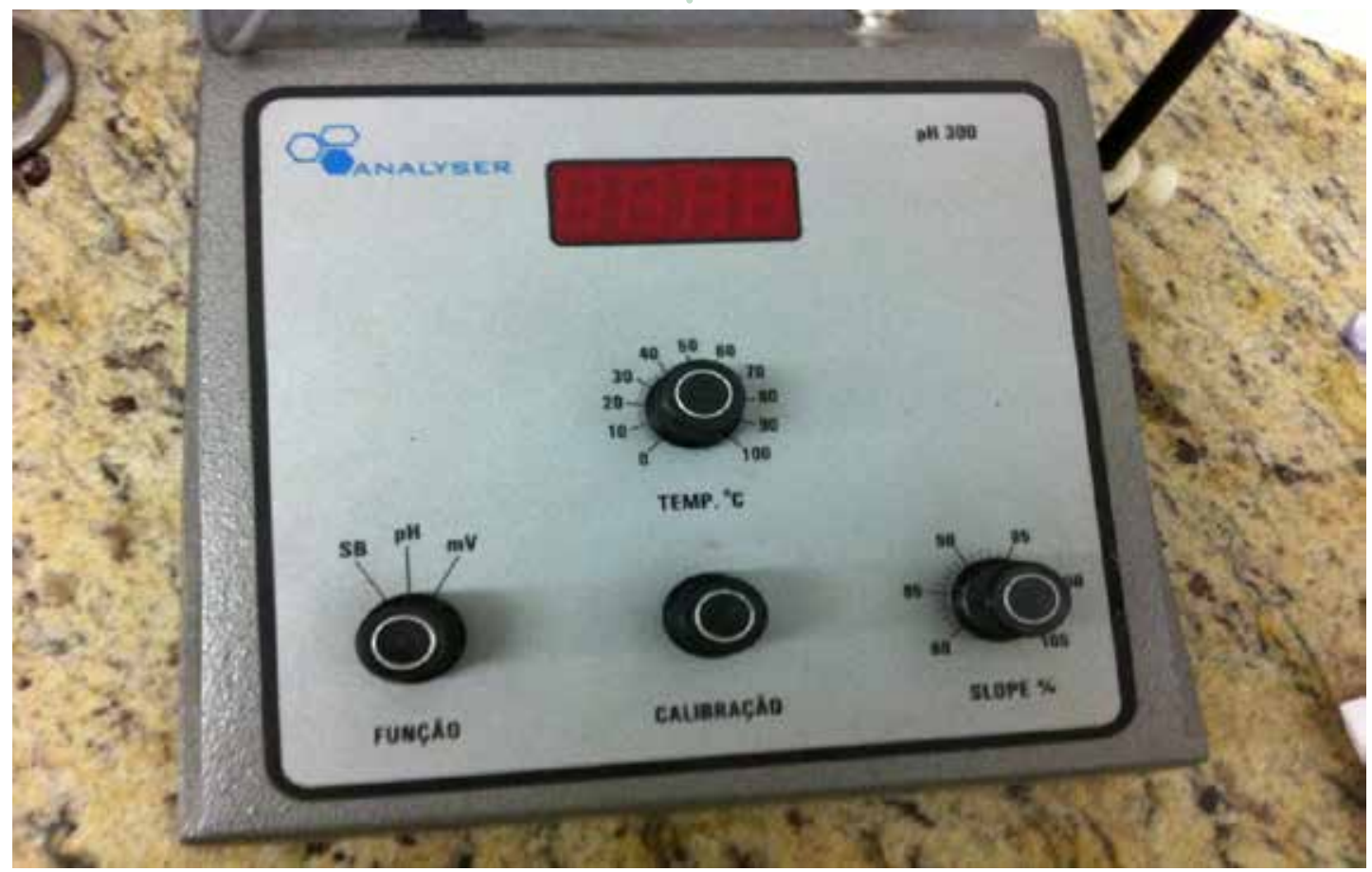

Figura 4 - Phmetro

PA 1,0011g associado à trietanolamina 2,4829g manipulado com uma consistência que permitiu o preenchimento com uma broca lentulo e selado com cimento provisório Cotosol marca VIGODENT.

$\mathrm{Na}$ sequencia, os dentes foram presos em um pote de armazenamento individual que foi preenchido com $5 \mathrm{ml}$ de água destilada com o pH em torno de 7. Em seguida, fez-se a leitura do $\mathrm{pH}$ nos tem- pos: $0 \mathrm{~h}, 24 \mathrm{~h}, 48 \mathrm{~h}, 72 \mathrm{~h}, 7$ dias, 10 dias, 15 dias e 30 dias, utilizando-se o pHmetro da marca ANALYSER, inserindo-se a sua ponta no interior do pote, dentro da água destilada.

Após a mensuração, com os dados anotados em uma tabela, foi realizada a análise estatística do $\mathrm{pH}$, aplicando o teste t de Student pareado, para comparar as médias de $\mathrm{pH}$ em cada tempo de avalia- 
ção das substâncias hidróxido de cálcio + anestésico e hidróxido de cálcio + trietanolamina. Para comparar a ação mais rápida em relação ao maior $\mathrm{pH}$ ao longo do tempo estudado, foi utilizado o teste ANOVA e, a posteriori, o teste de Tukey-Kramer. Para todos os testes foi adotado o nível de significância de 5\% $(\mathbf{\square}=0,05)$.

\section{RESULTADOS}

$\mathrm{O}$ teste $\mathrm{t}$ pareado indicou diferenças extremamente significativas $(\mathrm{p}<0,0001$; $\mathrm{t}$ $=47,089$ ) entre as médias de cada tempo estudado, no valor do $\mathrm{pH}$ da combinação hidróxido de cálcio + trietanolamina.

O teste ANOVA mostrou diferenças muito significativas $(p<0,0014 ; F=3,823)$ entre os valores de $\mathrm{pH}$ ao longo do tempo das substâncias médias de $\mathrm{pH}$ por tempo de estudo de hidróxido de cálcio + anestésico, principalmente, de acordo com o teste de Kruskal-Wallis, entre 0 hora e 15
RODRIGUES MCM RANGEL LFO

PEREIRA VFGC

CHAVES ES

SILVEIRA JCF

NORA MB

AVALIAÇÃO DO

PH EXTERNO

RADICULAR DO

HIDRÓXIDO DE

CÁLCIO ASSOCIADO

A DIFERENTES

FÁRMACOS

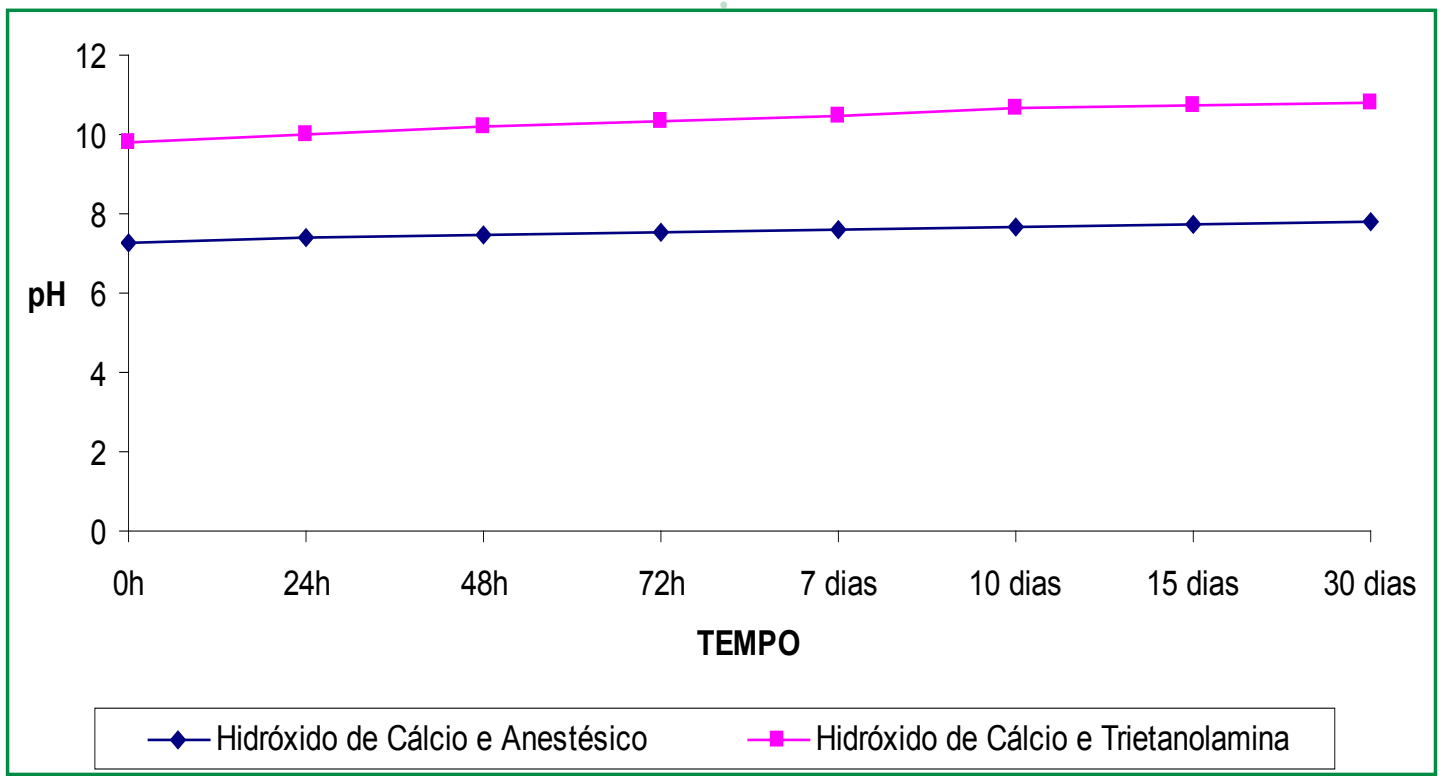

Figura 5 - Médias de pH por tempo de estudo de hidróxido de cálcio + anestésico e hidróxido de cálcio + trietanolamina

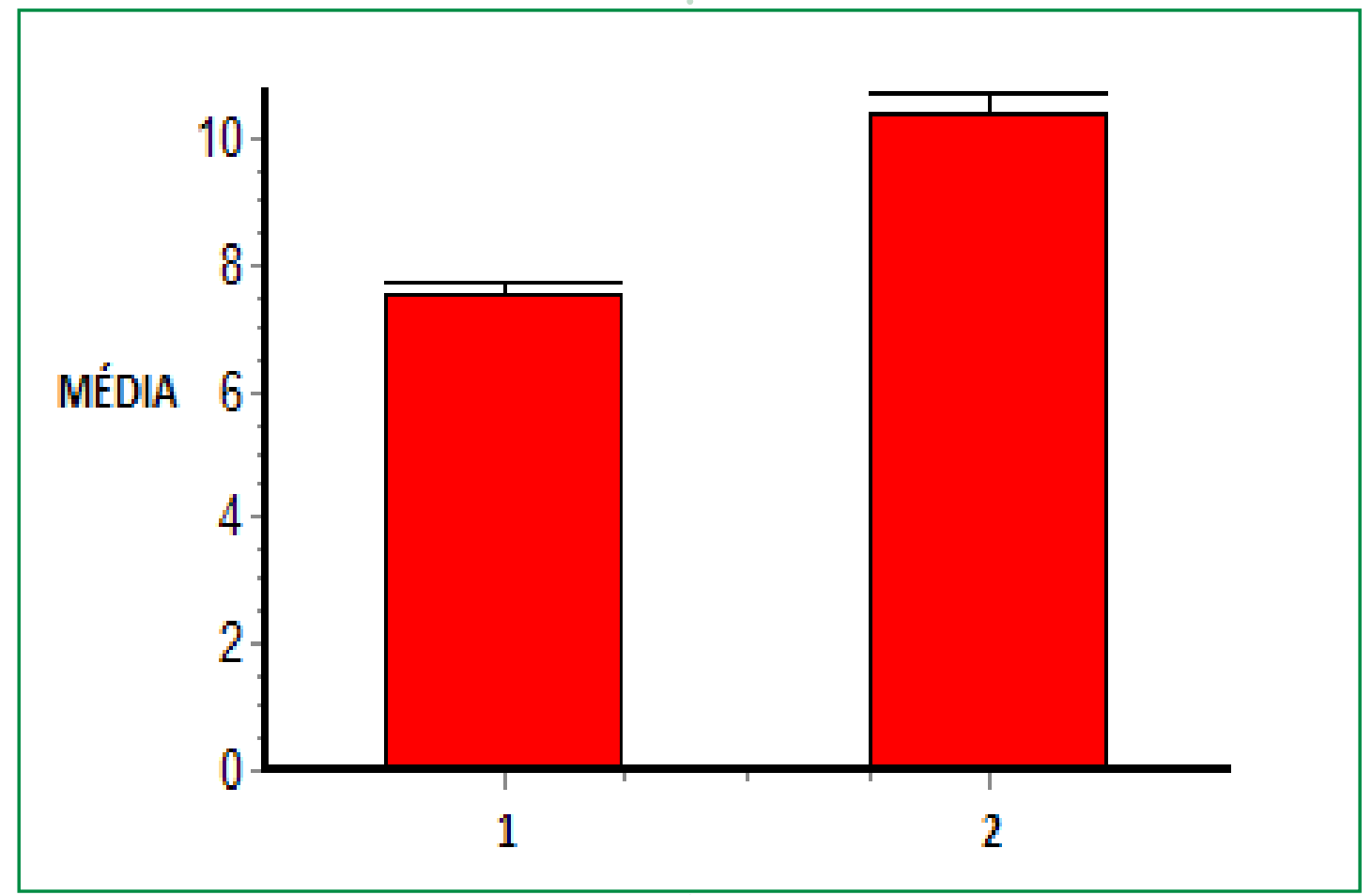

Figura 6 - Médias de pH de hidróxido de cálcio + anestésico (1) e hidróxido de cálcio + trietanolamina (2). Barras verticais indicam desvio-padrão.

REV, ODONTOL UNIV. CID, SÃO PAULO JAN - ABR 
RODRIGUES MCM RANGEL LFO

PEREIRA VFGC

CHAVES ES

SILVEIRA JCF

NORA MB

AVALIAÇÃO DO

PH EXTERNO

RADICULAR DO

HIDRÓXIDO DE

CÁLCIO ASSOCIADO

A DIFERENTES

FÁRMACOS

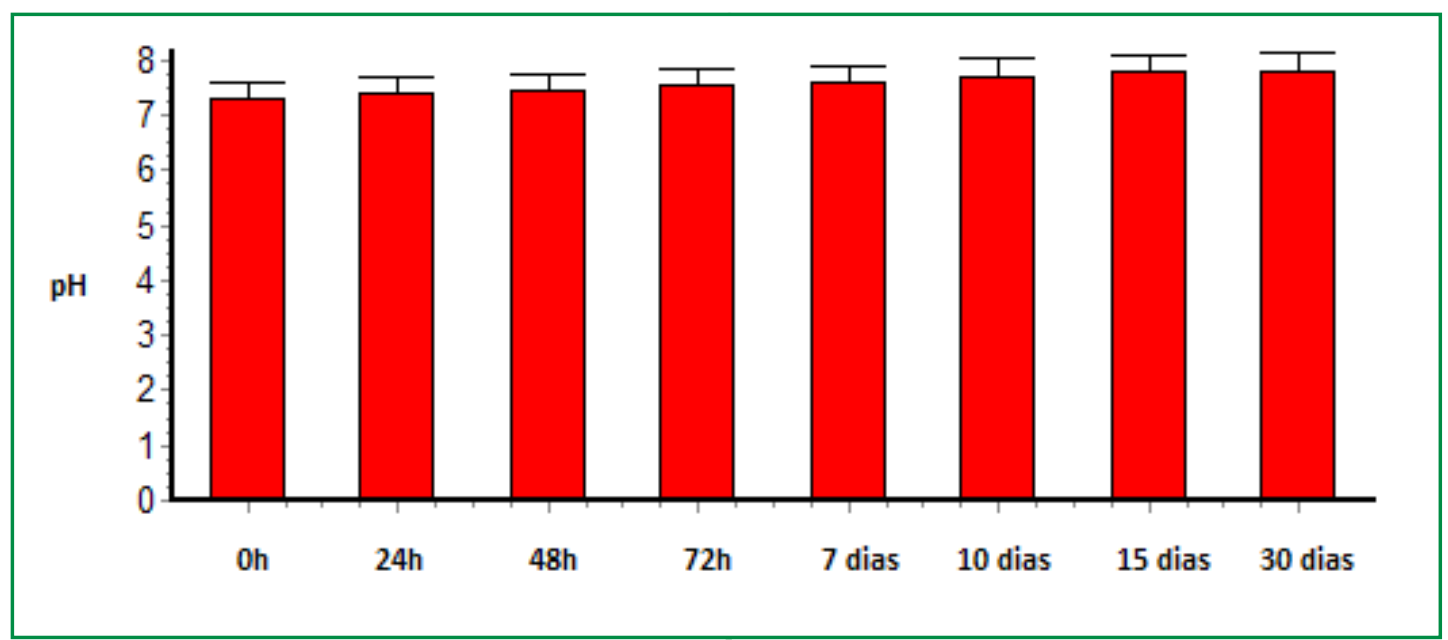

Figura 7 - Médias de $\mathrm{pH}$ das substâncias hidróxido de cálcio + anestésico. Barras verticais indicam desvio-padrão

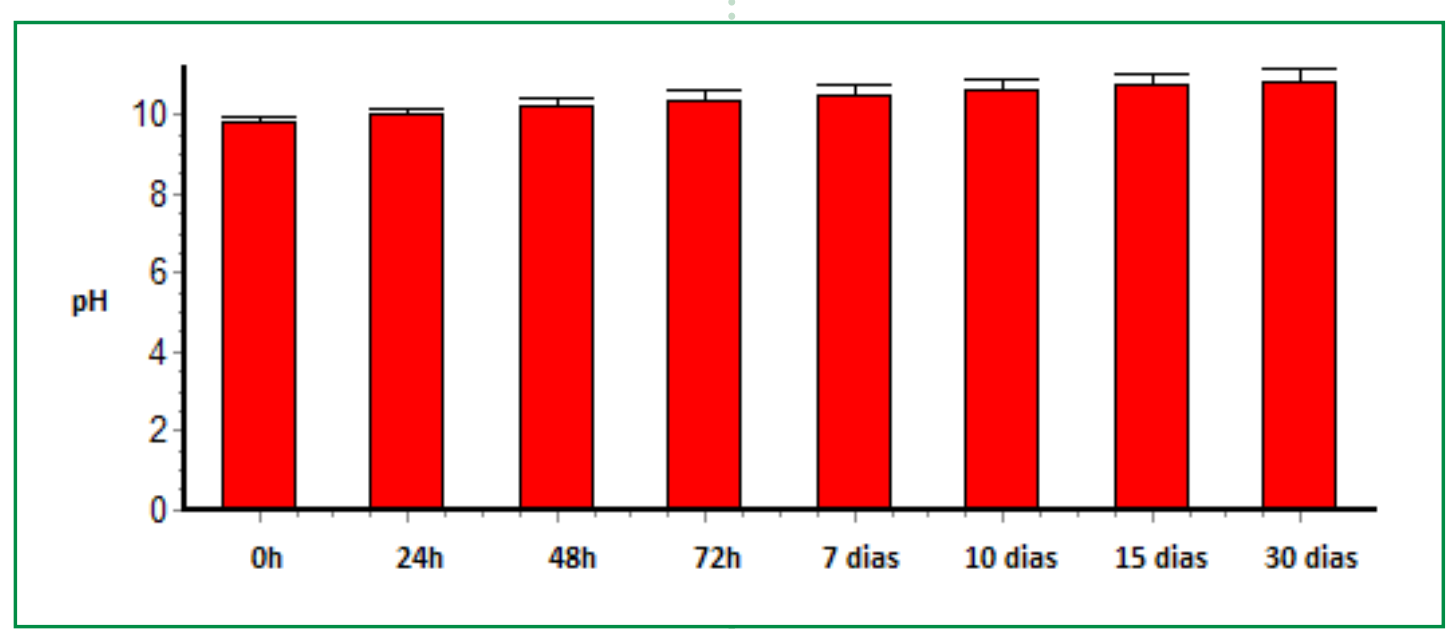

Figura 8 - Médias de pH das substâncias Hidróxido de cálcio + trietanolamina. Barras verticais indicam desvio-padrão

dias e 24 horas e 30 dias.

Em relação à variação do $\mathrm{pH}$ ao longo do tempo com as substâncias hidróxido de cálcio + trietanolamina, o teste ANOVA indicou diferenças extremamente significativas $(p<0,0001 ; F=22,628)$, e o teste de Kruskal-Wallis mostrou que as maiores diferenças estão entre 0 hora e 7 dias em diante e 24 horas e 7 dias em diante.

\section{I SCUSSÃO}

Pesquisas mostraram que, para o sucesso no tratamento endodôntico, precisa-se de uma boa instrumentação, irrigação, medicação intracanal e selamento dos canais ${ }^{2}$.

Com isso foi feito um estudo que mostrou que a adição de determinadas substâncias aos compostos à base de hidróxido de cálcio, com o propósito de oferecer condições para favorecer o uso clínico, podem modificar o $\mathrm{pH}^{3}$.

Diversos autores pesquisados concordaram em que o hidróxido de cálcio, quando utilizado puro ou em associações como uma medicação intracanal, promove ação antisséptica, antibactericida e antimicrobiana devido ao seu aumento no $\mathrm{pH}^{1,2,4,5,6,7,8}$. No entanto, Tavares et al. ${ }^{9}$ testaram e mostraram que o hidróxido de cálcio quando isolado, não apresenta ação antimicrobiana.

O hidróxido de cálcio associado a alguns veículos tem a possibilidade de alcalinizar o meio externo radicular, atuando sobre algumas bactérias existentes na cavidade oral ${ }^{4}$. Assim, alguns veículos foram pesquisados para se ter uma boa efetividade na medicação intracanal, veículos estes que são: água destilada, soro fisioló- 
gico, solução anestésica, trietanolamina, paramonoclorofenol, paramonoclorofenol canforado, óleo de girassol, polietilenoglicol, óleo de oliva, cerumin e digluconato de clorexidina; porém, existem discordâncias dentre as substâncias associadas ao hidróxido de cálcio para se analisar um melhor resultado $10,11,12,13,14,15,16,17,18,19$, $20,21,22,23,24$.

A proposição deste estudo foi avaliar o hidróxido de cálcio associado a diferentes fármacos, na tentativa de se achar uma substância que consiga extrair o máximo das propriedades dele, analisando-se qual veículo favorece a alcalinização do meio externo. Foram utilizados o anestésico que é o mais utilizado e a trietanolamina devido ao seu $\mathrm{pH}$ mais elevado.

Os resultados indicaram que o hidróxido de cálcio associado à trietanolamina teve melhor resultado do que a associação com o anestésico e notou-se que o pH teve um aumento ao longo do tempo independente do veículo utilizado.
Foi verificado em estudos que a associação com o anestésico promoveu uma estabilização com relação ao tempo e que o pH mostrou-se alcalino, justificando essa associação como uma boa medicação intracanal ${ }^{6,16,25,26,27,28}$. O resultado do presente trabalho entra em acordo com os trabalhos citados, apontando que foi encontrado um $\mathrm{pH}$ alcalino.

Em relação à trietanolamina, essa associação teve um aumento no $\mathrm{pH}$ no decorrer do tempo, mostrando um $\mathrm{pH}$ bem elevado, em concordância com outras pesquisas já realizadas ${ }^{14,24,29,30}$.

\section{CONCLUSÃO}

Os resultados obtidos através da metodologia empregada mostraram que o $\mathrm{pH}$ foi se elevando com o tempo, verificado com as duas substâncias. Houve uma variância entre as pastas testadas, indicando que o hidróxido de cálcio, associado à trietanolamina, teve um $\mathrm{pH}$ mais elevado do que quando associado ao anestésico.
RODRIGUES MCM

RANGEL LFO

PEREIRA VFGC

ChaVES ES

SILVEIRA JCF

NORA MB

AVALIAÇÃO DO

PH EXTERNO

RADICULAR DO

HIDRÓXIDO DE

CÁlCIO ASSOCIADO

A DIFERENTES

FÁRMACOS

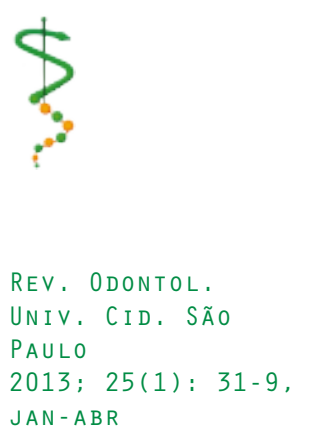


RODRIGUES MCM RANGEL LFO

PEREIRA VFGC CHAVES ES

SILVEIRA JCF NORA MB

AVALIAÇÃO DO PH EXTERNO RADICULAR DO HIDRÓXIDO DE CÁLCIO ASSOCIADO A DIFERENTES FÁRMACOS

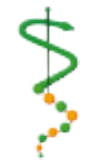

REV, ODONTOL.

UNIV, CID, SÃO

PAULO

2013; 25(1): 31-9,

$J A N-A B R$
REFERÊNCIAS

1. Siqueira Júnior JF, Lopes HP. Hidróxido de Cálcio em Endodontia: Suposições $x$ Comprovação Científica. Rev Bras Odontol. 1997; 54(4): 186-93.

2. Estrela C, Sydney GB, Bammann LL, Felippe Júnior O. Estudo do Efeito Biológico do Ph na Atividade Enzimática de Bactérias Anaeróbicas. Rev Fac Odontol Bauru - SP. 1994; 2(4): 31-8.

3. Leonardo MR, Reis RT, Silva LAB, Lofredo LCM. Hidróxido de Cálcio em Endodontia. RGO. 1992; 40: 69-72.

4. Esberard RM, Cames J, David L, Delrio CE. Reabsorção Radicular: Influência de Diferentes Tipos de Pasta de Hidróxido de Cálcio na Mudança do pH da Superfície Radicular. RGO. 1996; 44(5): 267-70.

4. Holland R, Otoboni Filho JA, Souza V, Nery MJ, Bernabé PFE, Dezan Júnior E. Reparação dos Tecidos Periapicais com Diferentes Reformulações de $\mathrm{Ca}(\mathrm{OH}) 2$ Estudado em Cães. Rev Assoc Paul Cir Dent. 1999; 53(4): 327-31.

5. Camargo CHR, Afonso SE, Valera MC, Mancini MNG, Bernardineli N, Oliveira LD. Avaliação do pH e Liberação de Íons de Cálcio na Utilização Intracanal das Pastas à base de Hidróxido de Cálcio. Cienc Odontol Bras. 2003; 6(1): 51-9.

6. Soares JA, Soares TRS. Ação Antisséptica de Pastas Intracanais à base de Hidróxido de Cálcio. Rev CROMG. 2003; 9(2): 94-100.

7. Oliveira EPM, Irala LED, Santos AR, Melo TAF. Avaliação da Ação Antimicrobiana de Quatro Formulações a Base de Hidróxido de Cálcio Utilizadas como Medicação Intracanal. RFO. 2010. 15(1): 35-9.

8. Tavares AR, Beurmer J, Pereira RR, Masiero AV. CFC: Uma Alternativa para as Infecções Endodônticas Resistentes?. Rev Bras Odontol. 2010; 67(1): 123-7.

9. Leonardo MR, Reis RS, Silva LAB, Assed S. Determinação de Íons Ca+2, pH e Solubilidade de Pastas à Base de Hidróxido de Cálcio Contendo PMC e PMCC. Rev Bras Odontol. 1993; 50(1): 5-10.

10. Siqueira Júnior JF, Magalhães FAC, Uzeda M. Avaliação Antibacteriana de Medicação Intracanal. R G O. 1996; 44(2): 271-4.

11. Kuga MC, Ito CM, Yamashita JC, Duarte MAH, Fayad MVL, Ogata M. Influência do Tipo de Pasta de Hidróxido de Cálcio e do Método de Irrigação Final no Vedamento de Obturações Endodônticas. F O L Unimep. 2000; 12(1-2): 57-61.

12. Sonoda CK, Poi WR, Barioni SRP, Okamoto T, Barbosa ALP. Influência da Pasta de Hidróxido de Cálcio no Reimplante Dental Mediato. Rev Bras Odontol. 2002; 59(4): 236-40.

13. Simi Júnior J. Avaliação Histológica da Biocompatibilidade da Pasta de Hidróxido de Cálcio Veiculada à Trietanolamina Através de Implantes Intraósseos em Guinea-pig. [Tese] 2003.

14. Alencar AH, Pimenta FC, Ito IY, Bruno KF, Leonardo MR. Determinação dos Microrganismos no Canal Radicular, Antes do Preparo Biomecânico e Após a utilização da Medicação Intracanal, em Dentes com Necrose Pulpar e reação Periapical Crônica. Arq Odontol - BH. 2005; 41(2): 105-92.

15. Estrela C, Estrela CRA, Guimarães LF, Silva RS, Pecora JD. Tensão do Hidróxido de Cálcio Associado a Diferentes Substâncias. J Appl Oral Sci. 2005; 13(2): 152-6.

16. Maniglia CAG, Picolli F, Spadaro ACC, Lima OJ, Maniglia AB. Dissociação lônica de Medicação Intracanais Experimentais à Base de Hidróxido de Cálcio. J Bras Endod. 2005; 5(21-22): 433-40. 
17. Nagem Filho H, Nagem HD, Coutinho KQ, Carvalho PRMA, Fiuza CT. Propriedades do Paramonoclorofenol Canforado e Paramonoclorofenol Canforado Associado ao Hidróxido de Cálcio. Pesq Bras Odontoped Clin Integr. 2007; 7(3): 235-9.

18. Maia Filho EM, Maia CCR, Bastos ACSC, Novais TMG. Efeito Antimicrobiano In Vitro de Diferentes Medicações Endodônticas e Própolis Sobre Enterococcus Faecalis. R G O. 2008; 56(1): 21-5.

19. Repeke HP, Westphalen VPD, Silva Neto UX, Carneiro E, Fariniuk LF, Sousa MH, Brancher JA. Estudo do pH de Três Diferentes Pastas de Hidróxido de Cálcio. Rev Clin Pesq Odontol. 2008; 4(3): 169-73.

20. Pereira L, Nabeshima CK, Britto MLB, Pallota RC. Avaliação do pH de Substâncias Utilizadas como Medicação Intracanal em Diferentes Veículos. R S B O. 2009; 6(3): 243-7.

21. Kuga MC, Pirolla MO, Freitas PC, Sant'Anna Júnior A, Guerriro-Tanomaru JM, Só MVR. Avaliação In Vitro do pH do Hidróxido de Cálcio Usado como Medicação Intracanal em Associação com Clorexidina e Racealfatocoferol. R F O. 2010; 15(2): $150-4$.

22. Pereira MJ, Parreira MLJ, Chavasco JK. Avaliação da Atividade Antimicrobiana In Vitro de Pastas à Base de Hidróxido de Cálcio. Rev Univ Vale do Rio Verde-Três Corações. 2011; 9(2): 328-36.

23. Rangel LFGO. Avaliação do pH Externo Radicular Após Utilização Intracanal de Associações de Hidróxido de Cálcio a Diferentes Veículos. [Mestrado] São Paulo: Universidade de Taubaté - São Paulo; 2012.

24. Rangel LFGO. Avaliação do pH Externo Radicular Após Utilização Intracanal de Associações de Hidróxido de Cálcio a Diferentes Veículos. [Mestrado] São Paulo: Universidade de Taubaté - São Paulo; 2012.

25. Lage-Marques JLS, Conti R, Antoniazzi JH, Gutz I. Avaliação da Velocidade de Dissociação lônica do Hidróxido de Cálcio Associado a Diferentes Veículos. Rev Odontol Univ Cid São Paulo. 1994; 8(2): 81-7.

26. Takeuti ML, Lage JLSM, Antoniazzi JH. Penetração Dentinária da Associação Hidróxido de Cálcio, Ciprofloxacina e Metronidazol Variando o Veículo. R G O. 1997; 4(2): 146-52.

27. Dotto SR, Travassos RMC, Ferreira R, Santos R, Wagner M. Avaliação da Ação Antimicrobiana de Diferentes Medicações Usadas em Endodontia. Rev Odontol Cienc. 2006; 21(53): 266-9.

28. Moura SU, Martos J, Silveira LFM. Análise da Formação de Carbonato de Cálcio pela Reação do Hidróxido de Cálcio com Diferentes Veículos Anestésicos. Pesq Bras Odontoped Clin Integr. 2008; 8(3): 271-6.

29. Simi Júnior J, Ishimoto MA, Silva AHFE, Ferrari CH, Medeiros JMF. Avaliação pela Análise Digital da Capacidade de Penetração Dentinária do Hidróxido de Cálcio Associada à Trietanolamina. Rev Pós-Graduação Facul Odontol São Paulo. 2000; 7: 16.

30. Figueiredo CMC. Estudo das Variações do pH das Pastas de Hidróxido de Cálcio Veiculada à Trietanolamina. [Monografia] Associação Brasileira de Odontologia de Campo Belo; 2003. 65p.

Recebido em: 22/11/2012

Aceito em: 04/12/2012 